\title{
Interdigitated Microelectrode Arrays Integrated in Microfluidic Cell for Biosensor Applications
}

\author{
Baccar $\mathrm{H}^{1 *}$, Mejri MB${ }^{1}$, Prehn $\mathrm{R}^{2}$, del Campo $\mathrm{FJ}^{2}$, Baldrich $\mathrm{E}^{3}$, Rosemonde $\mathrm{M}^{4}$ and Abdelghani $\mathrm{A}^{1}$ \\ ${ }^{1}$ Nanotechnology Group, INSAT, Bp676, Centre Urbain Nord, 1080 Charguia Cedex, Tunisia \\ ${ }^{2}$ Institute of Microelectronics of Barcelona IMB -CNM (CSIC ), Campus of the Autonomous University of Barcelona, 08193 - Bellaterra, Barcelona, Spain \\ ${ }^{3}$ Nanotools (Cibbim-Nanomedicine). Vall d'Hebron Institut de Recerca (VHIR). Passeig de la Vall d'Hëron, 119-129 - 08035 Barcelona, Spain \\ ${ }^{4}$ Biophage Pharma, 6100 Royalmount, Montreal, Canada
}

\begin{abstract}
In this work we present an approach for the use of phages as bioreceptors for bacteria detection in a microfluidic cell by Electrochemical Impedance Spectroscopy (EIS). With this aim, the biocomponent has been immobilized on interdigitated gold microelectrodes with the phyisorption technique. The three electrodes (reference electrode, counter electrode and work electrode) are integrated on the same chip and a microfluidic cell is used. We have shown that chip is stable in liquid medium for several hours. For the detection side, the interaction of E.coli bacteria with T4 phages shows an increase of the impedance. A detection limit of $10^{3} \mathrm{CFU} \mathrm{mL}{ }^{-1}$ was obtained with good reproducibility.
\end{abstract}

Keywords: Interdigitated gold microelectrodes; Microfluidic cell; T4-phage; Escherichia coli bacteria; Electrochemical impedance spectroscopy

\section{Introduction}

Since the 80's, localized outbreaks of infection have created a pervasive climate of anxiety, and the development of more and more frequent antibiotic resistance restores to the microorganisms, which are represented by various forms including bacteria, fungi, protozoa, the virus.... the potential morbid thought that they lost it. Hence, the need for a means for rapid detection of pathogens. At the crossroads of several disciplines such as physics, electronics, optics, mechanics, chemistry, and biology, nanotechnology is characterized by the manipulation of structures on the scale of the atom or molecule. Indeed, the miniaturization of existing techniques, such as chromatography, PCR, electrophoresis, ELISA, spectrophotometry and titration is very challenging. In addition, these techniques are expensive, difficult to implement and sample preparation and exploitation of results is often slow. In recent decades, research has intensified to develop new devices for rapid analysis that meet the needs of areas related to chemistry, biology, food and clinical research, hence the biosensors. Indeed, the recognition of the species by the sensitive element is accompanied by a change in physical properties easily measurable. The study of these biosensors is interesting because it allows both to reconcile the practical aspects of device development and basic research aspects concerning the study of interactions between species and to detect functional materials brought into play. Many transduction techniques are used to measure the interaction between the sensitive element and the element to detect.

Electrochemical Impedance Spectroscopy (EIS) is a powerful electrochemical technique capable of detecting small changes occurring at the solution-electrode interface. The electrochemical process at the surface of an electrode is associated with the production of charges during the contacting of a conductor with an electrolyte. Such transfer of charges is followed by a passage of electric current through the interface due to the appearance of an electric field. EIS is used as a technique for the characterization of the conductivity of the materials (wires, insulators) in liquid medium [1,2]. Recently, impedance spectroscopy is used to study the conductivity of biomembranes or self-assembled monolayers deposited on microelectrodes or nanodevices.

Several studies have been conducted by researchers using EIS for pathogen detection, monitoring the changes in the average conductivity caused by bacterial growth / metabolism, or the changes in the electrode-solution interface due to micro-organisms nonspecific or specific adsorption $[3,4]$.

Bacterial cells were immuno-sensed by EIS using interdigitated microelectrodes with detection limits of $10^{4} \mathrm{CFU} \mathrm{ml}{ }^{-1}$ for Escherichia coli and Salmonella typhimurium analyzed in saline solution [5-7]. In these works, bacteria captured on top of or between the electrode fingers behaved as insulator elements and negatively affected electron transfer. Detection in more complex environments, such as culture media or in the presence of non-target bacteria, generated a large background signal, attributed by the authors to non-specific biocomponent adsorbed on the sensor surface.

Mejri et al. and Shabani et al. $[3,8]$ proposed a unique alternative for EIS bacteria biosensing, based on the use of bacteriophages, instead of antibodies. Phage production is possible in an easier, faster, and cheaper way than antibody $(\mathrm{Ab})$ production, and does not depend on the use of animals, which makes them promising tools in biosensing $[9,10]$. In their work, they performed EIS measurements for bacteria detection using phages. Interestingly, they registered not only an increase in electrolyte resistance caused by the bacterial recognition by phages, but also a simultaneous decrease in charge-transfer resistance caused by the bacterial infection and lysis and the sensor had a reported detection limit of $10^{4} \mathrm{CFU} \mathrm{m}{ }^{-1}$ in saline buffer. But in their work, they were using an impedance analyser with a conventional one-compartment voltammetric cell with a three-electrode configuration separated.

We now go a step further; in this work, we miniaturized the

${ }^{*}$ Corresponding author: Hamdi Baccar, Nanotechnology Group, INSAT, Bp676, Centre Urbain Nord, 1080 Charguia Cedex, Tunisia, Tel: 216-717-03-829; E-mail: hamdibaccar@yahoo.fr

Received October 13, 2014; Accepted November 11, 2014; Published November 20, 2014

Citation: Baccar H, Mejri MB, Prehn R, del Campo RJ, Baldrich E, et al. (2014) Interdigitated Microelectrode Arrays Integrated in Microfluidic Cell for Biosensor Applications. J Nanomed Nanotechnol 5: 243. doi: 10.4172/2157-7439.1000243

Copyright: $\odot 2014$ Baccar H, et al. This is an open-access article distributed under the terms of the Creative Commons Attribution License, which permits unrestricted use, distribution, and reproduction in any medium, provided the original author and source are credited. 


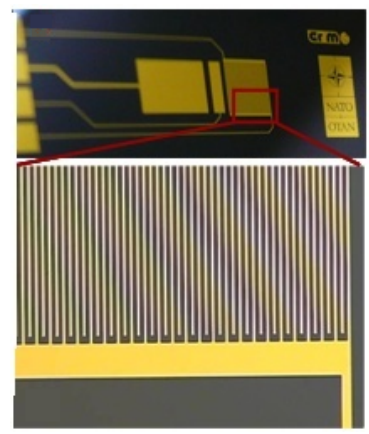

(A)

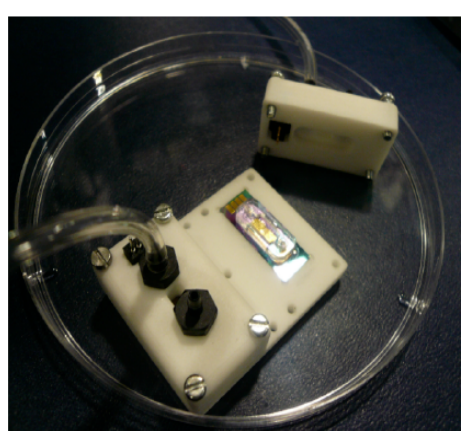

(B)
Figure 1: A) Interdigitated gold microelectrodes, B) Microfluidic cell.

biosensor using interdigitated microelectrodes in which the three electrodes are integrated on the same chip. Furthermore, a microfluidic cell with maximum volume of $50 \mu \mathrm{l}$ was used. The limit detection of bacteria was optimized.

\section{Materials and Methods}

\section{Interdigitated gold microelectrode micro fabrication}

Interdigitated microelectrodes were produced by standard photolithographic techniques and extensively characterised as described in references [11]. Briefly, a micron thick silicon oxide layer was thermally grown over a 4 -in. silicon wafer. Next, the electrodes were patterned by a lift-off technique. This consisted in the deposition of a photoresist over the wafer, which was then exposed through a suitable quartz-chromium mask. After developing the resist, the wafer was metallised by sputtering. This metal layer was composed by $10 \mathrm{~nm}$ titanium, $10 \mathrm{~nm}$ nickel and $100 \mathrm{~nm}$ gold. Nickel acts as diffusion barrier to avoid the formation of undesirable Ti-Au alloys. After the metallisation, the wafer was immersed in an acetone bath where the excess resist was removed, leaving the microelectrode structures neatly defined on the wafer. The next step consisted in depositing a passivation layer to protect the contact lines of the devices. This was a mixed layer of silicon oxide ( $4000 \AA$ ) and silicon nitride (7000 ̊). After this, the wafer was newly coated in photoresist and insulated through a second mask that defines the final electrode geometry and contact pads. After the resist was developed, it was hardened by soft bake in order to withstand the etching steps that followed. The passivation layer was etched in a reactive ion etching step, followed by a wet etching step that removed any traces of silicon oxide from the surface of the electrodes.

Last, the wafers were diced into $20 \mathrm{~mm} \times 7.5 \mathrm{~mm}$ individual chips, each chip features two interdigitated gold electrodes, each of them consisting of 50 fingers $10 \mu \mathrm{m}$ wide, separated $10 \mu \mathrm{m}$ from the nearest band. Similarly, a reference electrode (in gold) and a counter electrode (in gold) of larger area than the working electrode are integrated on the same microchip (Figure 1A).

Before their utilisation, the electrodes were electrochemically activated by applying series of potential pulses ( $10 \mathrm{~s}$ each) at 0 and $-2 \mathrm{~V}$ (versus reference electrode) in $0.5 \mathrm{M} \mathrm{NaNO}_{3}$ solution. The degree of activation was then verified by cyclic voltammetry in $1 \mathrm{mM}$ potassium ferrocyanide $\left(\mathrm{K}_{4} \mathrm{Fe}(\mathrm{CN})_{6}\right)$. A chip was considered active and suitable for impedance measurements when the cyclic voltammograms of its two electrodes were similar and the peak-to-peak separation in the voltammetry of a ferricyanide solution did not exceed $70-80 \mathrm{mV}$ at 50 $\mathrm{mVs}^{-1}$.

\section{Microfluidic cell}

The support is a microfluidic flow cell of small volume for detection in a liquid medium using microchips manufactured (Figure 1B). The system features two microchips simultaneously (for electrochemical measurements) and composed of two PDMS microfluidic channel each and two polyamide supports made by laser sintering in a Formiga P100 (EOS, DE). These parts are designed to fit and seal by the contact pressure, so that the bonding and encapsulation resin are avoided. This device was used for electrochemical measurements in parallel with a stream of liquid to flow. The pumping rate supported by this system is 1

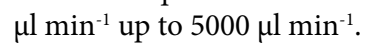

\section{Bacteria culture and T4-phage culture}

E.coli $\mathrm{K} 12$ and T4-phage culture were produced by standard techniques and extensively described [3]. Briefly, E. coli K12 cells, provided by BioPhage Pharma (Montreal, Canada), and was grown on liquid LB medium. Concentration was estimated after plating on solid LB medium. Boxes containing between 30 and 300 colonies are the only boxes that count in the counting step. Counting has led to a concentration during the stationary phase estimated by $10^{8} \mathrm{CFU} \mathrm{ml} l^{-1}$. The storage was performed at $-20^{\circ} \mathrm{C}$ by adding $10 \%$ glycerol. Viability and concentration batches stored were also verified by plating on solid LB medium. Then, the bacteria was diluted in sterile $\mathrm{PBS} \mathrm{pH}=7$ and ready for manipulation.

To cultivate these phages, bacteria specific to them were grown until exponential phase was reached, then they were inoculated with $500 \mu \mathrm{l}$ of phage. The suspension was incubated at $37^{\circ} \mathrm{C}$ in an incubator shaker for 3 hours. Chloroform was added and the solution was kept at $4^{\circ} \mathrm{C}$ for $20 \mathrm{~min}$, followed by an ultrafiltration through a sterile filter and centrifugation. The purified phages were resuspended in sterile PBS, serially diluted, and titrated. The phage stock produced in this way was at an approximate titter of $10^{7} \mathrm{PFU} \mathrm{ml}{ }^{-1}$.

\section{Bioreceptors physisorption}

For the modification of the gold surface by physisorption with our phages, $100 \mu \mathrm{l}$ of the solution of phage $\left(10^{7} \mathrm{PFU} \mathrm{ml}{ }^{-1}\right)$ were deposited directly on the microelectrodes, followed by incubation for $90 \mathrm{~min}$. Incubations were carried out inside a homemade humid chamber at $37^{\circ} \mathrm{C}$. After surface modification with the appropriate bioreceptor, the microelectrodes were subjected to intensive washing with sterile PBS. Subsequently, $100 \mu \mathrm{l}$ of a solution of BSA $(1 \%$, w/v) were deposited on microelectrodes modified and were incubated for $45 \mathrm{~min}$. This step is necessary to prevent blocking the non-specific adsorption of the biocomponent non-target.

\section{Electrochemical impedance spectroscopy}

The electrochemical measurements were performed at room temperature. We used an Autolab302N impedance analyser (Ecochemie, The Netherlands) equipped with the NOVA1.4 acquisition software. The impedance spectra were obtained in a frequency range from 50 $\mathrm{mHz}$ to $1 \mathrm{MHz}$, using a modulation voltage of $5 \mathrm{mV}$. All electrochemical measurements were carried out in sterile PBS and inside a Faraday cage. The PBS sterile solution contained $140 \mathrm{mM} \mathrm{NaCl}, 2.7 \mathrm{mM} \mathrm{KCl}, 0.1 \mathrm{mM}$ $\mathrm{Na}_{2} \mathrm{HPO}_{4}$, and $1.8 \mathrm{mM} \mathrm{KH}_{2} \mathrm{PO}_{4}$ and had a conductivity of $14.73 \mathrm{mS}$ $\mathrm{cm}^{-1}$. 


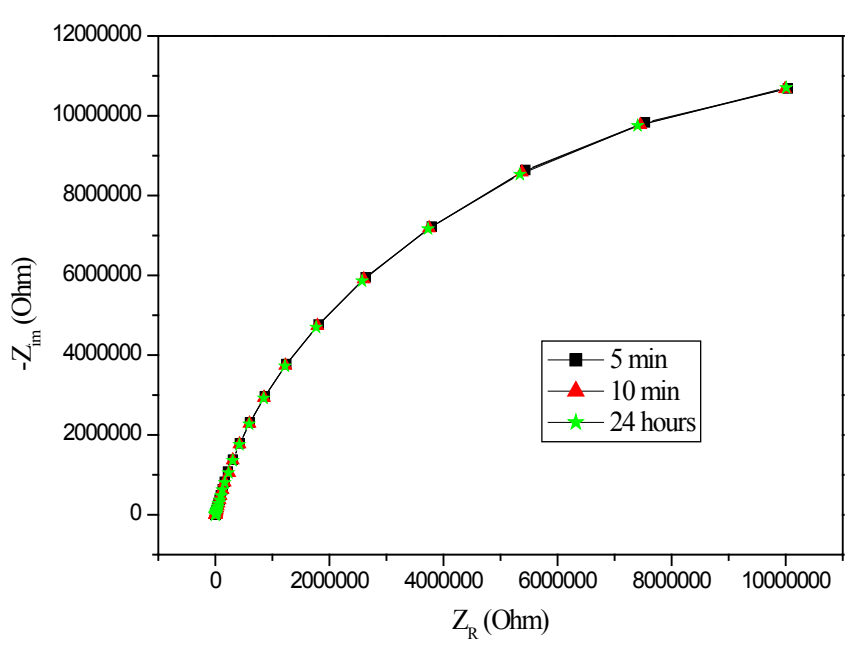

Figure 2a: Microelectrode stability over time.

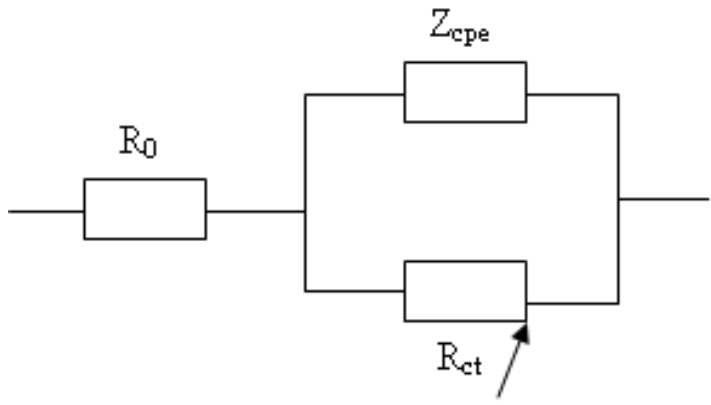

Figure 2b: Equivalent circuit of the interdigitated microelectrode/electrolyte interface.

\section{Results and Discussion}

\section{Electrochemical characterization of interdigitated gold microelectrodes}

The microelectrodes used in this work were produced by standard photolithographic techniques. The microelectrodes were manufactured with the three electrodes were integrated on the same chip. Work started with microelectrode characterization in order to test signal stability. The stability of interdigitated gold microelectrodes after activation was verified by electrochemical impedance spectroscopy.

Figure 2A shows the Nyquist diagram $(50 \mathrm{mHz}-1 \mathrm{MHz})$ of the chip in the microfluidic cell for different incubation times in a solution of PBS: $5 \mathrm{~min}, 10 \mathrm{~min}$ and 24 hours. We observed that the impedance spectrum of the microelectrode is stable even after a long period, which will give us reliable results thereafter. The electric model appropriate for this chip is presented in Figure $2 \mathrm{~B}$ where the resistance $\mathrm{R}_{0}$ is the resistance of the electrolyte at high frequency, $R_{c t}$ corresponds to the resistance of charges transfer at the chip/electrolyte and $Z_{C P E}$ is the impedance of constant phase element.

\section{Bacteria EIS biosensing at microelectrodes modified with phages}

We next studied the response over time of gold microelectrodes, modified phages and blocked with BSA as previously described, in the presence of $E$. coli. A remarkably different behaviour is observed at phage-modified microelectrodes. Figure 3 shows the variation in the impedance $(\Delta \mathrm{z})$ before and after the injection of the concentration $10^{5}$ CFU ml-1 of the bacteria recorded at $233 \mathrm{mHz}$ as a function of time for a microelectrode modified with phage T4. The curve shows the presence of two signals, an initial increase in impedance which lasts about 70-75 min, followed by a short period of stabilization signal and a decrease in impedance. The initial increase is due to the signal recognition phage - bacteria [12]. The decrease in impedance happens probably by phageinduced bacterial infection and lysis, which induces cell wall disruption and release of important amounts of intracellular components. This behavior was consistently observed for all the chips studied and in all the experiments performed. Also, as observed by Shabani et al. [8], this event induces increase of the medium conductivity at the vicinity of the electrode surface, which contributes to decrease charge-transfer resistance and the results obtained are similar to the results obtained by Mejri et al. [3] except the fact of working at low volume, increases the time of analysis by electrical measurement but this time is always less than the time taken by conventional techniques (PCR, ELISA).

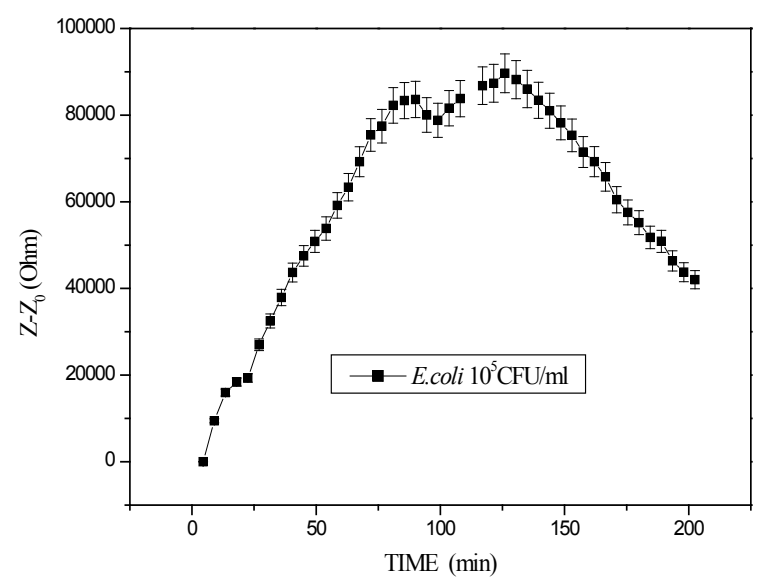

Figure 3: The impedance variation as a function of time for the T4 phage in the presence of a bacteria concentration of $10^{5} \mathrm{CFU} / \mathrm{ml}$.

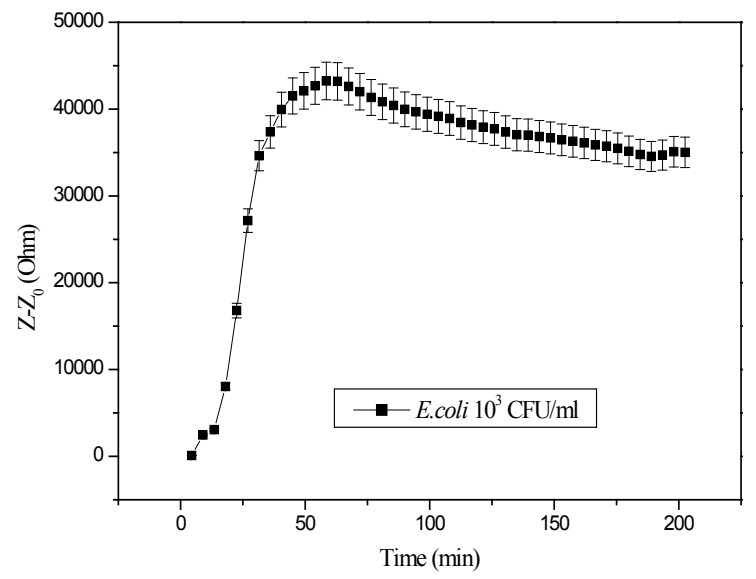

Figure 4: The impedance variation as a function of time for the T4 phage in the presence of a bacteria concentration of $10^{3} \mathrm{CFU} / \mathrm{ml}$. 


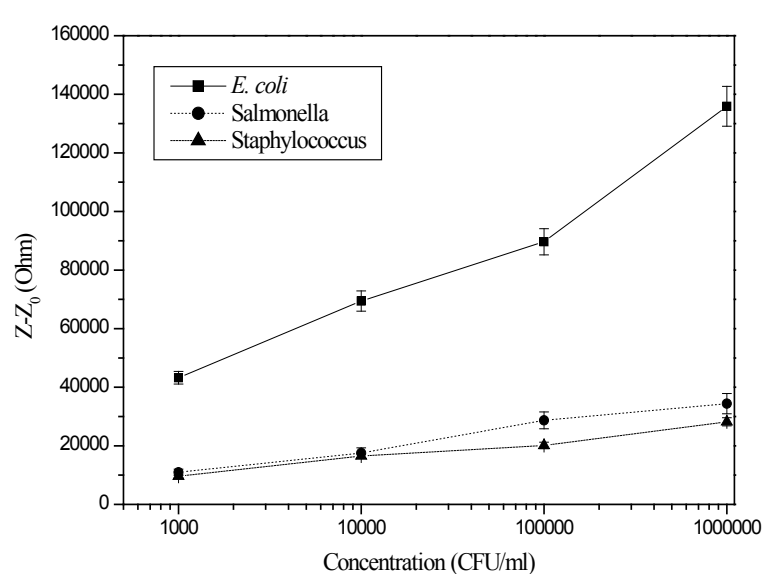

Figure 5: Calibration curves of (a) E. coli as the Specific test and (b) Salmonella as the non-specific test and (c) Staphylococcus as the nonspecific test.

In order to optimize the detection limit, another concentration of E. coli was injected for detection. Figure 4 illustrates the impedance variation versus time for phage T4 immobilized on a microelectrode in the presence of a concentration $10^{3} \mathrm{CFU} \mathrm{ml}{ }^{-1}$ of E. Coli. This time the behavior is slightly different from the one obtained for the concentration $10^{5} \mathrm{CFU} \mathrm{ml}{ }^{-1}$. An increase of the signal and a slow decrease can be explained by the detection limit.

Figure 5 shows the calibration curves obtained for different concentration of E. coli (specific test) and for Salmonella (non-specific test) and for Staphylococcus (non-specific test). As we can see, the increase of the impedance is due to specific recognition of E.coli bacteria with phages T4. It shows a linear response with detection limit of $10^{3}$ $\mathrm{CFU} \mathrm{ml}{ }^{-1}$ and with sensitivity equal to $27.210 \mathrm{KOhm} \mathrm{CFU}^{-1}$.

\section{Conclusion}

In the present work, we present an innovative alternative which comprises a system for bacterial detection based on the use of phages as bioreceptors by Electrochemical Impedance Spectroscopy (EIS). We have shown that the microelectrodes are stable in the microfluidic cell in liquid medium. It has been shown also that phages can be adsorbed on the surface of the gold microelectrode by physisorption. Phages are easy to produce and their preparation is inexpensive. A detection limit of $10^{3} \mathrm{CFU} \mathrm{ml}{ }^{-1}$ was obtained with good reproducibility.

\section{Acknowledgment}

This research is sponsored by NATO's Public Diplomacy Division in the Framework of "Science for Peace", project SFP 983115. The authors thank Biophage Pharma (Canada) for providing E. coli and T4 phages.

\section{References}

1. Baccar H, Mejri MB, Hafaiedh I, Ktari T, Aouni M, et al. (2010) Surface plasmon resonance immunosensor for bacteria detection. Talanta 82: 810-814.

2. Mejri MB, Baccar H, Ktari T, Aouni M, Abdelghani A (2011) Detection of E.Coli Bacteria Using Impedance Spectroscopy and Surface Plasmon Resonance Imaging Based Biosensor. Sensors Letters 9: 2130-2132.

3. Mejri MB, Baccar H, Baldrich E, Del Campo FJ, Helali S, et al. (2010) Impedance biosensing using phages for bacteria detection: generation of dual signals as the clue for in-chip assay confirmation. Biosens Bioelectron 26: 1261-1267.

4. Varshney M, Li Y (2009) Interdigitated array microelectrodes based impedance biosensors for detection of bacterial cells. Biosens Bioelectron 24: 2951-2960.

5. Zourob M, Elwary S, Turner APF (2008) Principles of Bacterial Detection Biosensors, Recognition Receptors, and Microsystems. Springer Science \& Business Media.

6. Radke SM, Alocilja EC (2005) A high density microelectrode array biosensor for detection of E. coli O157:H7. Biosens Bioelectron 20: 1662-1667.

7. Laczka O, Baldrich E, del Campo FJ, Muñoz FX (2008) Immunofunctionalisation of gold transducers for bacterial detection by physisorption. Bioanal Chem 391 : 2825-2835.

8. Shabani A, Zourob M, Allain B, Marquette CA, Lawrence MF, et al. (2008) Bacteriophage-modified microarrays for the direct impedimetric detection of bacteria. Anal Chem 80: 9475-9482.

9. Lakshmanan RS, Guntupalli R, Hu J, Kim DJ, Petrenko VA, et al. (2007) Phage immobilized magnetoelastic sensor for the detection of Salmonella typhimurium. J Microbiol Methods 71: 55-60.

10. Balasubramanian S, Sorokulova IB, Vodyanoy VJ, Simonian AL (2007) Lytic phage as a specific and selective probe for detection of Staphylococcus aureus-A surface plasmon resonance spectroscopic study. Biosens Bioelectron 22 948-955.

11. Laczka O, Baldrich E, Muñoz FX, del Campo FJ (2008) Detection of Escherichia coli and Salmonella typhimurium using interdigitated microelectrode capacitive immunosensors: the importance of transducer geometry. Anal Chem 80: 72397247.

12. Singh A, Poshtiban S, Evoy S (2013) Recent advances in bacteriophage based biosensors for food-borne pathogen detection. Sensors (Basel) 13: 1763-1786. 